\title{
Diabète et obésité : qu'avons-nous appris de l'étude des Indiens Pimas?
}

\section{Jean-François Gautier Éric Ravussin}

J.F. Gautier : Service de diabétologie et d'endocrinologie, Hôpital Saint-Louis, 1, avenue Claude-Vellefaux, 75475 Paris Cedex 10, France. E. Ravussin : Pennington Biomedical Research Center, Louisiana State University, 6400 Perkins Road, Bâton Rouge, Louisiana 70808-4124, États-Unis.

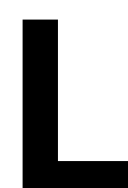

es Indiens Pimas vivent dans le désert Sonoran au sud de l'Arizona depuis au moins 2000 ans. Leurs ancêtres lointains ont fait partie de la première migration paléo-indienne à travers le détroit de Bering il y a 30000 ans. On pense qu'ils descendraient des Hohokam qui ont migré du Mexique vers la Gila River (désert Sonoran) environ 300 ans avant Jésus Christ. Les Hohokam ont apporté lors de leur migration une connaissance approfondie de l'agriculture qu'ils ont adaptée au désert en construisant des systèmes d'irrigation sophistiqués. Ainsi, ils ont su transformer la terre aride du désert en ferme et produire du maïs, des haricots et du coton. Les systèmes d'irrigation étaient riches de poissons et attiraient le gibier, ce qui améliorait l'alimentation des Indiens Pimas. A la fin du XIX $x^{\mathrm{e}}$ siècle, les colons venus de l'est se sont appropriés l'eau des rivières pour leur propre agriculture asséchant ainsi bon nombre de fermes des Indiens Pimas. La carence en eau a transformé l'économie et le style de vie des Indiens. Ils sont devenus dépendants des aides gouvernementales qui leur apportaient du sucre, du lard et de la farine de mil. Ainsi, le passage au XXe siècle a été marqué par la perte de l'activité physique liée aux travaux des champs et le changement brutal de leur alimentation [1].

\section{Prévalence du diabète de type 2 et de l'obésité}

Au début du $x x^{e}$ siècle, le diabète était rare parmi les Indiens Pimas [2]. En 1963, une étude de prévalence de la polyarthrite rhumatoïde dans cette population révélait que de nombreux membres de la communauté étaient diabétiques. Au vu des résultats, le $\mathrm{NIH}$ (National Institutes of Health) mettait en route deux ans plus tard une étude prospective au sein de la communauté d'Indiens Pimas vivant dans la réserve de la Gila River. Tous les deux ans, chaque résident de plus de cinq ans est convié pour un examen comprenant la mesure du poids et de la taille et une épreuve d'hyperglycémie provoquée par voie orale (75g de glucose). A partir de cette étude qui est toujours en cours, il a été montré que les Indiens Pimas sont hyperinsulinémiques et résistants à I'insuline comparativement à d'autres groupes ethniques. Par ailleurs, la prévalence du diabète de type 2 et de l'obésité est très élevée dans cette 
population (m/s 1999, $n^{\circ} 10$, p. 1187).

\section{Prévalence du diabète de type 2}

La forte prévalence du diabète de type 2 devient évidente dans la seconde moitié du $\mathrm{Xx} \mathrm{x}^{\mathrm{e}}$ siècle. Le diabète de type 2 est considéré comme un problème de santé majeur dans la communauté au cours des années 1960 et sa prévalence ne cesse d'augmenter depuis cette époque. Plus de la moitié des adultes âgés de plus de 35 ans sont atteints de diabète. La figure 1 représente la prévalence du diabète de type 2 chez les Indiens Pimas, hommes et femmes, âgés de 20 à 74 ans, comparée à celle observée dans la population blanche des États-Unis [3].

Le diabète de type 2 est caractérisé par des anomalies métaboliques majeures: une obésité, une diminution de l'action de l'insuline, une anomalie de la sécrétion de l'insuline et une augmentation de la production hépatique de glucose.

Depuis 1982, des Indiens Pimas présentant une tolérance normale au glucose ont été admis au Centre de recherches cliniques du $\mathrm{NIH}$, environ tous les ans, afin de mesurer l'action de l'insuline à l'aide d'un clamp euglycémique hyperinsulinémique

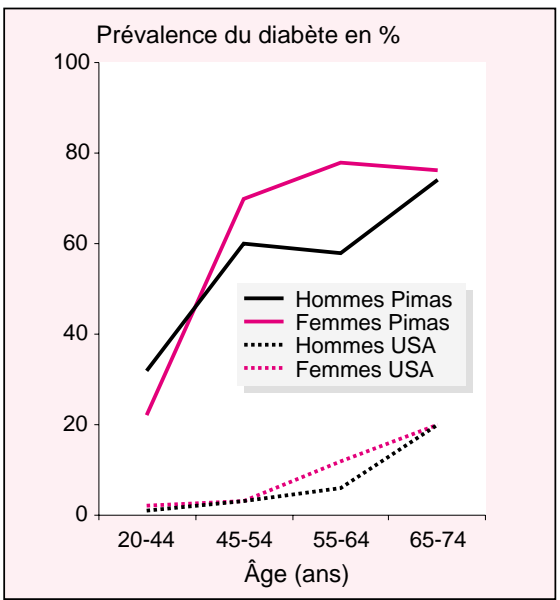

Figure 1. Prévalence du diabète de type 2 en fonction du sexe et de l'âge chez les Indiens Pimas étudiés entre 1981 et 1988, comparativement à celle observée dans la population caucasienne des États-Unis (d'après [7]). (méthode de référence pour évaluer la sensibilité à l'insuline) et la sécrétion de l'insuline au cours d'une hyperglycémie provoquée par voie intraveineuse. Trois anomalies métaboliques se sont révélées des prédicteurs de la survenue du diabète de type 2: I'obésité, l'insulinorésistance et une diminution de l'insulinosécrétion en réponse au glucose [4]. Récemment, il a été montré que ceux qui développent un diabète dans les 5 ans présentent une détérioration progressive de l'action de l'insuline et une augmentation du poids au cours du temps [5]. La phase précoce de I'insulinosécrétion diminue lors du passage de la tolérance normale au glucose vers l'intolérance au glucose et s'effondre lors du passage de l'intolérance au glucose vers le diabète. L'augmentation de la production hépatique de glucose à jeun est un phénomène tardif puisqu'elle n'est observée que lors du passage de l'intolérance au glucose vers le diabète. Ceux qui ont conservé une tolérance normale au glucose pendant cette période ont également pris du poids et aggravé la résistance à l'insuline mais la phase précoce de l'insulinosécrétion a augmenté parallèlement à la diminution de l'action de I'insuline [5]. Ainsi, une diminution de l'action de l'insuline et de la sécrétion de l'insuline en réponse au glucose sont des phénomènes précoces dans la physiopathologie du diabète de type 2 .

\section{Prévalence de l'obésité}

La définition de l'obésité - un état $\mathrm{d}^{\prime}$ excès de masse grasse corporelle est relativement arbitraire et fondée sur une approximation de la graisse corporelle, l'indice de masse corporelle (BMI ou body mass index = poids en $\mathrm{kg} /$ taille $^{2}$ en $\mathrm{m}^{2}$ ). Un indice de masse corporelle $\geq 30 \mathrm{~kg} / \mathrm{m}^{2}$ est habituellement utilisé comme critère pour définir l'obésité dans les deux sexes bien que les femmes aient davantage de masse grasse que les hommes à BMI égal [6]. Les Indiens Pimas adultes sont en moyenne plus obèses que le reste de la population des États-Unis (figure 2). Entre 25 et 44 ans, plus de $64 \%$ des hommes et plus de $73 \%$ des femmes ont un BMI $\geq 30 \mathrm{~kg} / \mathrm{m}^{2}$. L'obésité chez les Indiens Pimas est un caractère fami-

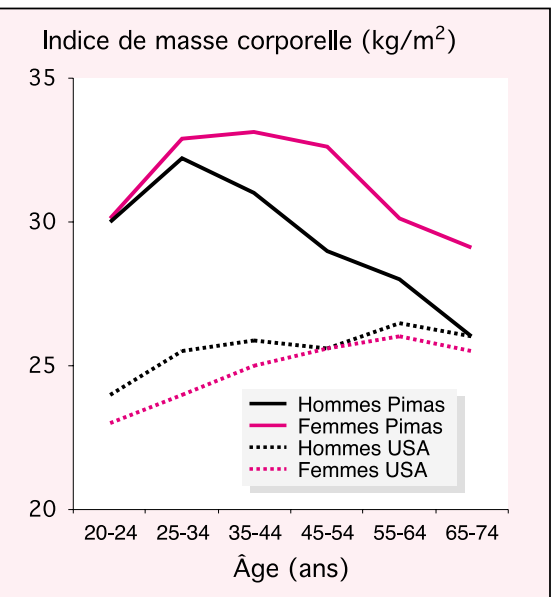

Figure 2. Indice de masse corporelle moyen en fonction de l'âge et du sexe chez les Indiens Pimas étudiés entre 1981 et 1988 (d'après [7]).

lial [7] et prédit le développement du diabète de type 2 . Cependant, une fois diabétique, les sujets tendent à perdre du poids [7]. La contribution relative des facteurs environnementaux et génétiques dans l'étiologie de I'obésité reste controversée.

Obésité: rôle de l'environnement

Toutes les études réalisées chez les jumeaux permettent de conclure qu'une grande partie de la variance du poids et de la composition corporelle est attribuée à des facteurs génétiques [8-10]. Cependant, puisque ces études ont été réalisées dans des pays industrialisés dans lesquels l'environnement est relativement homogène, caractérisé par une nourriture abondante, il est possible qu'elles aient sous-estimé le rôle de l'environnement.

Le rôle de l'environnement dans le développement de l'obésité est suggéré par l'association inverse, entre l'obésité et le statut socio-économique ou le niveau d'activité physique $[11,12]$. Par ailleurs, il existe une association positive entre obésité et temps passé devant la télévision [13], obésité et nombre de grossesses [12] et entre obésité et arrêt du tabac [12]. Enfin, les études de migration montrent que les populations à mode de vie traditionnel qui migrent dans des pays industrialisés présentent une 
augmentation de la prévalence de I'obésité et du diabète de type 2 [14, 15].

L'alimentation jouerait un rôle majeur dans la physiopathologie de l'obésité. Dans la majorité des pays industrialisés, I'alimentation "traditionnelle» pauvre en graisses, provenant de plantes non transformées, a été remplacée par une alimentation riche en graisses animales et en glucides simples, et pauvre en fibres. Ces modifications alimentaires se sont accompagnées d'une augmentation de la prévalence de l'obésité et des maladies cardiovasculaires [16]. La communauté $d^{\prime}$ Indiens Pimas d'Arizona qui présente la plus forte prévalence d'obésité et de diabète de type 2 rapportée dans le monde, illustre bien ce bouleversement de l'alimentation de "subsistance» vers une alimentation «industrialisée», bouleversement effectué sur une courte période. L'augmentation de la prévalence de l'obésité, parallèlement à ce changement de mode de vie, suggère qu'en présence d'une forte susceptibilité génétique - le génotype de "l'épargne » par exemple [17] I'environnement est un facteur causal de l'épidémie d'obésité et de diabète de type 2 observée dans cette population. Pour tester cette hypothèse, les Indiens Pimas d'Arizona ont été comparés aux Indiens Pimas ayant gardé un mode de vie traditionnel en ne participant pas à la migration vers le désert Sonoran. Ces Indiens Pimas sont restés localisés dans une région reculée et montagneuse située au Nord-Ouest du Mexique, région appelée "Pimeria»[1]. Jusqu'en 1991, I'accès à la communauté (village Maycoba) nécessitait 8 à 12 heures de transport avec un véhicule tout terrain à partir de Hermosillo (environ $160 \mathrm{~km}$ ). Depuis 1992, il existe une route pavée et étroite qui a raccourci la durée du trajet à 56 heures. Les Indiens vivant à Maycoba cultivent de manière traditionnelle (à la faux) des petites parcelles de terre à flanc de coteau (maïs, haricots, pommes de terre). Ils font pousser dans les jardins de leurs habitations des légumes tels que tomates, oignons, haricots verts... Ils élèvent du bétail. D'autres activités comprennent les travaux dans les ranchs, des constructions de route et des activités minières. La comparaison des Indiens
Pimas de chaque communauté a été réalisée en sélectionnant pour chaque Indien ( $\mathrm{n}=35$ ) vivant à Maycoba, 10 Indiens vivant en Arizona ayant le même âge et le même sexe. Les Indiens Pimas de Maycoba avaient un indice de masse corporel (BMI) plus bas $(24,9 \pm 4,0$ contre 33,4 \pm $\left.7,5 \mathrm{~kg} / \mathrm{m}^{2} ; \mathrm{p}<0,0001\right)$ et une cholestérolémie totale plus basse $(1,46 \pm$ 0,30 contre $1,75 \pm 0,31 \mathrm{~g} / \mathrm{l}, \mathrm{p}<$ $0,0001)$ comparativement à ceux vivant en Arizona. Par ailleurs, la prévalence du diabète de type 2 était nettement inférieure chez les Indiens Pimas de Maycoba, $11 \%$ contre $37 \%$ et $6 \%$ contre $54 \%$, respectivement chez les femmes et les hommes. Une étude récente ciblant toute la population adulte (plus de 200 individus) a établi que la prévalence du diabète de type 2 est de $6,4 \%$ dans cette population. Bien que les Indiens Pimas vivant à Maycoba avaient un poids plus bas que ceux vivant en Arizona, le BMI était de 17 à $36 \mathrm{~kg} / \mathrm{m}^{2}$ suggérant que même dans un environnement homogène, il existe une importante variabilité de poids. Ces données montrent que malgré une prédisposition génétique à prendre du poids, un mode de vie traditionnel caractérisé par une alimentation réduite, difficile à obtenir, moins riche en graisses animales et plus riche en glucides complexes, et par une activité physique plus importante, réduit le développement de I'obésité et du diabète de type 2 .

\section{Prédicteur métabolique de la prise de poids}

Chez les Indiens Pimas d'Arizona, il a été possible de mettre en évidence de manière prospective des facteurs de risque de développer une obésité (Tableau I).

\section{Dépenses énergétiques au repos}

L'obésité est associée avec une augmentation de la dépense d'énergie absolue à la fois au repos et sur les 24 h [18, 19]. Ainsi I'obésité ne peut pas être attribuée à une diminution du métabolisme de base comme cela a été proposé. Cependant, le graphique de corrélation entre la dépense énergétique et le poids corporel suggère que, pour un poids donné, des sujets peuvent avoir une dépense énergétique relative « haute», "normale» ou «basse ». La question de savoir si une dépense énergétique relative basse augmente le risque de prise de poids a été étudiée chez les Indiens Pimas d'Arizona. Une diminution relative de la dépense énergétique de base ou des 24 h, ajustée pour la masse maigre, la masse grasse, I'âge et le sexe, représente un facteur de risque pour la prise de poids ultérieure dans cette population [20]. Cependant, une diminution de la dépense énergétique relative ne représentait que $40 \%$ de la prise de poids et la dépense énergétique était devenue normale, si ce n'est haute, correspondant au nouveau poids des individus. Cette question a été étudiée dans d'autres populations. Roberts et al. [21] ont mesuré la dépense énergétique par la technique de l'eau doublement marquée chez 18 nouveau-nés âgés de 3 mois. A 12 mois, ceux qui étaient classés en surcharge pondérale avaient une dépense énergétique à 3 mois qui était de $20 \%$ inférieure à celle observée chez les bébés sans surcharge pondérale. De même, Griffith et al. [22] ont rapporté qu'une dépense énergétique basse chez des filles âgées de 5 ans était corrélée de manière négative avec l'indice de masse corporelle à I'adolescence. Ces données suggèrent que des traitements pharmacologiques qui augmentent la dépense énergétique pourraient être utiles dans le traitement de I'obésité, particulièrement chez l'individu présentant un métabolisme de base abaissé.

\section{Activité physique}

L'activité physique spontanée représente $8 \%$ à $15 \%$ de la dépense énergétique quotidienne totale [18]. Les études réalisées chez les Indiens Pimas montre que l'activité physique spontanée, mesurée dans une chambre calorimétrique, est un caractère familial et qu'elle est associée, lorsqu'elle est basse, à une augmentation de la prise de poids chez les hommes, mais pas chez les femmes [23]. Ces résultats sont en accord avec la plupart des études transversales qui ont montré que I'obésité était associée à une diminution de l'activité physique spontanée. Des études qui mesurent I'activité 
Tableau I. Facteurs de risque métaboliques associés à l'obésité: études transversales contre études longitudinales.

\begin{tabular}{|lcc|}
\hline & $\begin{array}{c}\text { Études transversales } \\
\text { (association à l'obésité) }\end{array}$ & $\begin{array}{c}\text { Études lonaitudinales } \\
\text { (prédiction de la prise de poids) }\end{array}$ \\
\hline $\begin{array}{l}\text { Dépense énergétique de repos } \\
\text { Dépense énergétique liée à l'activité physique }\end{array}$ & $\begin{array}{c}\text { Normale ou haute } \\
\text { Normale }\end{array}$ & Basse \\
Oxydation lipidique & Normale ou haute & Basse \\
Sensibilité à l'insuline & Basse & Haute \\
Activité du système nerveux sympathique & Haute & Basse \\
Concentration plasmatique de leptine & Haute & Basse \\
\hline
\end{tabular}

physique en vie «libre», par exemple en utilisant la technique de l'eau doublement marquée, sont nécessaires pour confirmer qu'un faible niveau d'activité physique spontané est un facteur de risque d'obésité.

\section{Oxydation lipidique}

La contribution relative des différents substrats énergétiques (lipides, protéines, glucides) dans I'alimentation, représente probablement un facteur important dans la physiopathologie de l'obésité. Ainsi, il a été rapporté que certains obèses ont une préférence pour les graisses alimentaires et consomment plus de lipides. La mesure des échanges gazeux par calorimétrie indirecte (consommation d'oxygène et production de gaz carbonique) associée à la mesure de I'azote urinaire permet de déterminer le quotient respiratoire (consommation $\mathrm{d}^{\prime} \mathrm{O}_{2} /$ production de $\mathrm{CO}_{2}$ ) non protéique $d^{\prime}$ un sujet. Ce quotient respiratoire est un reflet du ratio de l'oxydation des glucides et les lipides. La valeur est d'environ 0,8 lorsque les graisses sont le principal substrat oxydé (situation à jeun), et avoisine 1 lorsque I'oxydation glucidique est majoritaire (après un repas riche en glucides par exemple). Le quotient respiratoire non protéique dépend non seulement de la composition de la prise alimentaire, mais également de la balance énergétique (par exemple une balance énergétique négative entraîne une augmentation de l'oxydation des lipides), de l'adiposité de l'individu (une forte adiposité est associée à une augmentation de l'oxydation lipidique) et de I'appartenance familiale, suggérant des déterminants génétiques. Dans une étude récente, Toubro et al. [24] ont montré que les taux d'oxydation de substrats évalués par le quotient respiratoire représentaient un trait familial après ajustement pour la balance énergétique, le sexe et l'âge. Chez les Indiens Pimas, le quotient respiratoire des $24 \mathrm{~h}$ est également un trait familial et varie de manière importante parmi les individus [25]. Ceux qui avaient un quotient respiratoire dans le $90^{\mathrm{e}}$ percentile («faible oxydateur de lipides ») avaient 2,5 fois plus de risque de grossir de plus de 5 kilos comparés à ceux dont le quotient respiratoire était situé dans le $10^{\mathrm{e}}$ percentile (« oxydateur lipidique important»). Cet effet était indépendant de la dépense énergétique des $24 \mathrm{~h}$. Des résultats similaires ont été retrouvés chez des Caucasiens participant à la Baltimore Longitudinal Study on Aging [26]. De même, des études récentes ont montré que les patients qui ont été obèses ont un quotient respiratoire élevé (c'est-à-dire un faible taux d'oxydation lipidique) $[27,28]$ alors que ceux qui sont capables de maintenir leur perte de poids, ont un quotient respiratoire plus bas comparativement à ceux qui ont repris du poids [29].

\section{Sensibilité à l'insuline}

La sensibilité à I'insuline évaluée en mesurant I'utilisation totale du glucose stimulée par une perfusion d'insuline représente la somme de l'oxydation des glucides et du stockage des glucides sous forme de glycogène. Puisqu'un quotient respiratoire élevé (augmentation de l'oxydation des glucides) prédit la prise de poids, Swinburn et al. [30] ont étudié de manière prospective chez les Indiens Pimas si la sensibilité à l'insuline était associée à une prise de poids. Les sujets qui avaient une sensibilité à l'insuline située dans le $90^{\mathrm{e}}$ percentile avaient 3 à 4 fois plus de chance de grossir de plus de 10 kilos, comparés à ceux situés dans le $10^{\mathrm{e}}$ percentile (donc plus insulinorésistants). Cet effet était relié à l'oxydation du glucose et non au stockage. Ainsi, dans cette population, la sensibilité à l'insuline est un facteur de prise de poids alors que l'insulinorésistance est associée à une diminution de la prise de poids. Des résultats similaires ont été rapportés chez les Mexicains américains [31] et chez les Caucasiens de la San Luis Valley Diabetes Study [32]. En réponse à la prise de poids, la sensibilité à l'insuline diminue.

\section{Activité du système nerveux sympathique}

Les études réalises chez les Caucasiens montrent que l'activité du système nerveux sympathique est reliée à 3 composantes majeures de la dépense énergétique des $24 \mathrm{~h}$ : le métabolisme de repos, la thermogenèse post-prandiale et l'activité physique spontanée [33]. L'activité du système nerveux sympathique pourrait donc avoir un rôle dans la balance énergétique. Les Indiens Pimas ont une activité du système nerveux sympathique musculaire basse par rapport aux Caucasiens appariés par l'âge et par le poids [34]. De manière prospective (3 ans de suivi en moyenne), il a été montré que l'excrétion urinaire de noradrénaline (qui reflète l'activité du système nerveux sympathique) était corrélée négativement avec la prise de poids chez les hommes [35]. 
En conclusion, une diminution de I'activité du système nerveux sympathique est associée au développement de l'obésité.

\section{Concentration plasmatique de leptine}

La leptine provenant du gène $O B$ est une hormone sécrétée par le tissu adipeux qui inhibe la prise alimentaire et augmente la dépense énergétique [36]. Afin d'évaluer si les concentrations plasmatiques de leptine pouvaient jouer un rôle dans la prise de poids, les concentrations plasmatiques de leptine à jeun ont été mesurées chez deux groupes d'Indiens Pimas appariés pour le poids. Le groupe d'Indiens Pimas qui ont pris du poids pendant les 3 ans de suivi (en moyenne 23 kilos) avaient des concentrations de leptine initiales (ajustées à la masse grasse initiale) plus basses comparativement au groupe de sujets qui ont conservé un poids stable [37]. Ces données suggèrent que les concentrations plasmatiques de leptine relatives basses peuvent jouer un rôle dans le développement de l'obésité chez l'homme.

\section{Conclusions}

L'étude des Indiens Pimas, une communauté d'amérindiens marquée par une forte prévalence d'obésité et de diabète de type 2 , a apporté des progrès considérables dans la connaissance de la physiopathologie de ces deux maladies. Elle a permis de mettre en évidence l'interaction entre le rôle de l'environnement et celui de la génétique. Cela a été rendu possible grâce à la coopération active des Indiens Pimas avec le NIH localisé à Phoenix (Arizona, USA). Les facteurs métaboliques prédisposant à la prise de poids sont vraisemblablement génétiquement déterminés et semblent ne jouer qu'un rôle limité dans le développement de l'obésité. D'autres facteurs agissant sur la prise alimentaire pourraient avoir un rôle plus important. La prise alimentaire est un comportement qui pourrait $d^{\prime}$ ailleurs avoir une origine génétique. Le fait que des populations vivant dans des environnements différents ont une telle différence de poids corporel, souligne l'importance de
I'approche comportementale dans le traitement de l'obésité, fondée sur une diminution des calories (provenant en particulier des graisses) et une augmentation de l'activité physique. Tout le problème de cette approche thérapeutique est d'obtenir des sujets obèses le maintien permanent des modifications de comportement malgré leur préférence (naturelle) pour les graisses (génétiquement déterminée?). Seules des études prospectives à grande échelle permettront de confirmer l'efficacité de ce type d'approche thérapeutique

\section{RÉFÉRENCES}

1. Ravussin E, Valencia M, Esparza J, Bennett $P$, Schulz $L$. Effects of traditional lifestyle on obesity in Pima Indians. Diabetes Care 1994; 9: 1067-74.

2. Joslin EP. The university of diabetes. JAMA 1940; 115: 203-8.

3. Knowler WC, Pettitt DJ, Saad MF, Bennett $\mathrm{PH}$. Diabetes mellitus in the Pima Indians: incidence, risk factors and pathogenesis. Diabetes Metab Rev 1990; 6:1-27.

4. Lillioja S, Mott D, Spraul M, et al. Insulin resistance and insulin secretory dysfunction as precursors of non-insulin-dependent diabetes mellitus. N Engl J Med 1993; 30: 1988-92.

5. Weyer C, Bogardus C, Mott D, Pratley E. The Natural history of insulin secretory dysfunction and insulin resistance in the pathogenesis. J Clin Invest 1999; 104: 787-94.

6. Pérusse L, Chagnon YC, Rice T, Rao DC, Bouchard C. L'épidémiologie et la génétique moléculaire de l'obésité. Med Sci 1998; 14 : 914-24.

7. Knowler WC, Pettitt DJ, Saad MF, et al. Obesity in the Pima Indians. It magnitude and relationship with diabetes. Am J Clin Nutr 1991; 53: S1543-51.

8. Stunkard AJ Harris IR, Pederson NL, McClearn GE. The body-mass index of twins who have been reared apart. N Engl J Med 1990; 322: 1483-7.

9. Stunkard AJ, Sorensen TI, Hanis C, et al. An adoption study of human obesity. N Engl J Med 1986; 314: 193-8.

10. Bouchard C. Genetics of obesity. Ann Arbor: CRC Press, 1994

11. Rosenbaum M, Leibel RL, Hirsch J. Obesity. N Engl J Med 1997; 337 : 396-407.

12. Rissanen $A M$, Heliovaara $M$, Knekt $P$, Reunanen A, Aromaa A. Determinants of weight gain and overweight in adult finns. Eur J Clin Nutr 1991 ; $45: 419-30$.

13. Prentice AM, Jebb SA. Obesity in britain, gluttony or sloth? Br Med J 1995; 311 : 437-9.
14. Stern MP, Gonzales C, Mitchell BD, Villapado E, Haffner SM, Hazuda HP. Genetic and environmental determinants of type 2 diabetes in Mexico City and San Antonio. Diabetes 1992; 41: 484-92.

15. Oppert JM, Rolland-Cachera MF. Prévalence, évolution dans le temps et conséquences économiques de l'obésité. Med Sci $1998 ; 14: 939-43$.

16. WHO. Obesity: preventing and managing the global epidemic. Geneva: World Health Organization, 1997: 1-276.

17. Neel JV. Diabetes mellitus: a "thrifty» genotype rendered detrimental by 'progress »? Am J Hum Genet 1962; 14: 35362.

18. Ravussin E, Lillioja S,Anderson TE, Christin L, Bogardus C. Determinations of 24-hour energy experditure in man. J Clin Invest 1986; 78 : 1568-78.

19. Leibel RL, Rosenbaum M, Hirsch J. Changes in energy expenditure resulting from altered body weight. $N$ Engl J Med 1995; 332: 621-8.

20. Ravussin E, Lillioja S, Knowler WC, et al. Reduced rate of energy expenditure as a risk factor for the body weight gain. N Engl J Med 1988; 318: 467-72.

21. Roberts SB, Savage J, Coward WA, Chew B, Lucas A. Energy expenditure and intake in infants born to lean and overweight mothers. Lancet 1990; 318: 461-6.

22. Griffith M, Payne PR, Stunkard AJ, Rivers JPW, Cox M. Metablic rate and physical development in children at risk of obesity. Lancet 1990; 336: 76-8.

23. Zurlo F, Ferraro RT, Fontvieille AM, Rising R, Bogardus C, Ravussin E. Spontaneous physical activity and obesity; crosssectional and longitudinal studies in Pima Indians. Am J Physiol 1992; 263: E296-300.

24. Toubro $S$, Sorensen TIA, Hindsberger $C$, Christensen NJ, Astrup A. Twenty-four-hour respiratory quotient: the role of diet and familial resemblance. J Clin Endocrinol Metab 1998; 8: 2758-64.

25. Zurlo F, Lillioja S, Esposito-Del Puente $\mathrm{A}$, et al. Low ratio of fat to carbohydrate oxidation as predictor of weight gain: study of 24h RQ. Am J Physiol 1990; 259: E650-7.

26. Seidell JC, Muller DC, Sorkin JD, Andres $\mathrm{R}$. Fasting respiratory exchange ratio and resting metabolic rate as predictors of weight gain: Baltimore longitudinal study of aging. In J Obesity 1992; 16: 667-74.

27. Astrup A, Buemann B, Christensen NJ, Toubro S. Failure to increase lipid oxidation in response to increasing dietary at content in formerly obese women. Am I Physiol 1994; 266: E592-9.

28. Larson DE, Ferraro RT, Robertson DS, Ravussin E. Energy metabolism in weightstable post-obese individuals. Am J Clin Nutr $1995 ; 62$ : 735-9. 


\section{RÉFÉRENCES}

29. Froidevaux $F$, Schulz $Y$, Christin L, Jequier E. Energy experditure in obsese women before and during weight loss, after refeeding and in the weight-relapse period. Am J Clin Nutr 1993; 57:35-42.

30. Swinburn BA, Nyomba BL, Saad MF, et al. Insulin resistance associated with lower rates of weight gain in Pima Indians. J Clin Invest 1991; 88: 168-73.

31. Valdez R, Mitcheell BD, Hafffner SM, et al. Predictors of weight change in a bi-ethnic population: the San Antonio heart study. Int Obesity 1994; 18: 85-91.

32. Hoag S, Marshall JA, Jones RH, Hamman RF. High fasting insulin levels associated with lower rates of weight gain in persons with normal glucose tolerance: the San Luis Valley study. Int J Obesity 1995; 19: $175-80$.

33. Ravussin E, Gautier JF. Metabolic predictors of weight gain. Int J Obesity 1999 ; 23 (suppl 1) : 37-41.

34. Spraul M, Ravussin E, Fontvielle AM Rising R, Larson DE, Anderson EA. Reduced sympathetic nervous activity: a popential mechanism predisposing to body weight gain. J Clin Invest 1993; 92: 1730-5.

35. Tataranni PA, Young JB, Bogardus $C$ Ravussin E. A Low sympathoadrenal activity is associated with body weight gain and development of central adiposity in Pima Indian men. Obes Res 1997; 5 : 341-7.

36. Caro JF, Sinha MK, Kolaczinski JW Zhang PL. Considene RV Leptin: the tale of an obesity gene. Diabetes 1997; 45: 145562 .

37. Ravussin E, Pratley RE, Maffei M, et al Relatively low plasma leptin concentrations preced weight gain in Pima Indians. Nat Med 1997; 3 : 238-40.

\section{$\mathbf{m} \mathbf{S} 2000$ \\ Summary}

Diabetes and obesity

among the Pima Indians

More than half of the Pima Indians over 35 years of age have type 2 diabetes mellitus and more than $75 \%$ are overweight or obese. The Pima Indians have been the focus of prospective epidemiologic and metabolic studies for over three decades. Based on results from these studies, metabolic predictors of both diseases have been identified. Obesity, insulin resistance and low acute insulin secretion response to glucose have been demonstrating to be metabolic predictors of type 2 diabetes. For obesity, a low-relative metabolic rate, a low-fat/carbohydrate oxidation, a high insulin sensitivity, a low sympathetic nervous system activity and a relative low plasma leptin concentration are risk factors for the development of the disorder. This knowledge should provide information about candidate genes and improve chances of finding susceptibility genes for the diseases. In this manuscript, we have reviewed the history of the Pima Indians as well as the epidemiological studies on prevalence of type 2 diabetes and obesity. We also present data collected in a population with Pima ancestry in Northern Mexico which emphasize the impact of environment on the development of obesity and diabetes. Finally, in the last section, we review the metabolic risk factor for body weight gain in the Pima Indian population.

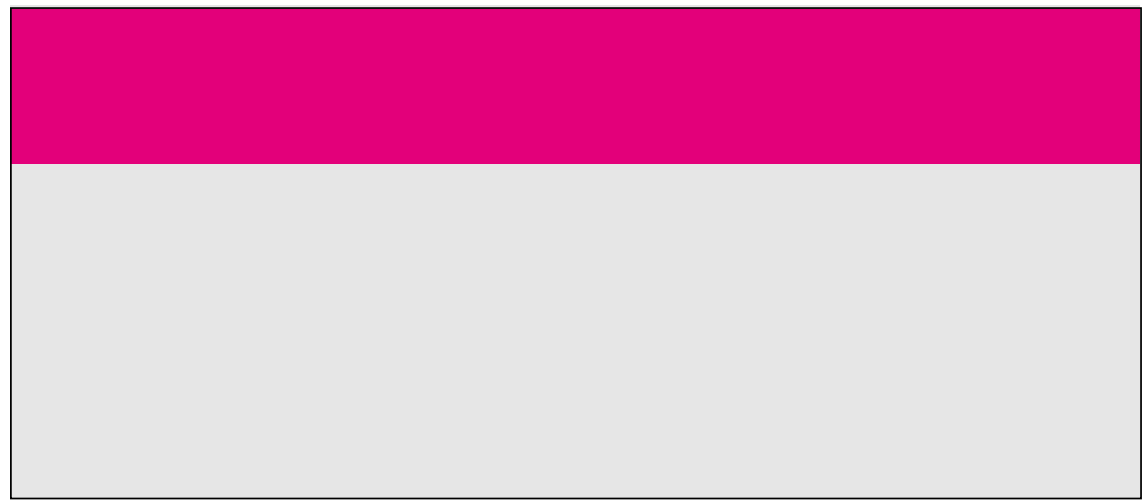

\section{Société Française d'Immunologie}

\section{Le douzième cours annue Immunologie : actualités 2000 Centre de congrès des Pensières, Annecy}

Organisation du cours : immersion totale du vendredi après-midi au mardi midi. Les conférenciers assistent à l'intégralité du cours.

Public: enseignants d'immunologie, jeunes scientifiques, chercheurs et ingénieurs.

Programme : xénogreffes, auto-immunité, peptides antimicrobiens, évolution du système immunitaire, complément, dynamique lymphocytaire, réaction allergique.

\section{Le congrès annuel Maison de I'UNESCO 29 novembre au 1er décembre 2000}

Le programme couvre les domaines suivants : bases moléculaires de la reconnaissance immunitaire, développement du système immunitaire, activation et fonctions effectrices, immunologie médicale, vaccins et immunothérapie. Des points d'actualité et des conférences de prestige seront également présentés. Enfin, le congrès est suivi d'une journée technologique le samedi 2 décembre 2000 sur le thème : techniques d'évaluation de l'apoptose.

\section{Renseignements}

La SFI est un organisme formateur agréé $n^{\circ} 11.75 .28 .994 .75$

Vous pouvez donc faire prendre en charge ces cours et congrès par votre organisme.

SFI, 28, rue du Dr-Roux 75724 Paris Cedex 15. Tél. : 0145688164 ou 0145668597

Fax : 0145674698 